\title{
Dielectric properties of lignin and glucomannan as determined by spectroscopic ellipsometry and Lifshitz estimates of non-retarded Hamaker constants
}

\author{
Rebecca Hollertz, Hans Arwin, Bertrand Faure, Yujia Zhang, Lennart Bergström and Lars \\ Wagberg
}

\section{Linköping University Post Print}

\section{Tweet}

N.B.: When citing this work, cite the original article.

The original publication is available at www.springerlink.com:

Rebecca Hollertz, Hans Arwin, Bertrand Faure, Yujia Zhang, Lennart Bergstrom and Lars Wagberg, Dielectric properties of lignin and glucomannan as determined by spectroscopic ellipsometry and Lifshitz estimates of non-retarded Hamaker constants, 2013, Cellulose (London), (20), 4, 1639-1648.

http://dx.doi.org/10.1007/s10570-013-9980-9

Copyright: Springer Verlag (Germany)

http://www.springerlink.com/?MUD=MP

Postprint available at: Linköping University Electronic Press http://urn.kb.se/resolve?urn=urn:nbn:se:liu:diva-100041 


\section{Dielectric properties of lignin and}

2 glucomannan as determined by

3 spectroscopic ellipsometry and Lifshitz

4 estimates of non-retarded Hamaker constants

5 Rebecca Hollertz ${ }^{\mathrm{a},{ }^{*}, \text { Hans Arwin }}{ }^{\mathrm{b}}$, Bertrand Faure ${ }^{\mathrm{c}}$, Yujia Zhang ${ }^{\mathrm{d}}$, Lennart

6 Bergström ${ }^{\text {c, e }}$, Lars Wågberg ${ }^{\text {a, e,* }}$

${ }^{a}$ Division of Fibre Technology, School of Chemical Science and Engineering,

9 KTH Royal Institute of Technology, SE-10044, Stockholm, Sweden

$10{ }^{b}$ Laboratory of Applied Optics, Department of Physics, Chemistry and Biology,

11 Linköping University, SE-58183, Linköping, Sweden

$12{ }^{c}$ Department of Materials and Environmental Chemistry, Arrhenius Laboratory,

13 Stockholm University, SE-106 91 Stockholm, Sweden

$14{ }^{d}$ Division of Wood Chemistry and Pulp Technology, School of Chemical Science and Engineering, KTH Royal Institute of Technology, SE-10044, Stockholm,

16 Sweden

$17{ }^{e}$ The Wallenberg Wood Science Centre, School of Chemical Science and

18 Engineering, KTH Royal Institute of Technology, SE-10044, Stockholm, Sweden

$19 *$ Corresponding authors:

20 Email: rhollert@kth.se, Phone: +4687908296, Fax: +4687906166

21 Email: wagberg@kth.se, Phone: +4687908294, Fax: +4687906166

We present in this study a quantitative estimate of the dispersive interactions between lignin, hemicellulose and cellulose, which are highly abundant materials in plants and trees. Spectroscopic ellipsometry was used to determine the dielectric properties in the UV-visible region of well-defined model materials of pure lignin and glucomannan. The non-retarded Hamaker constants were estimated from the determined spectral parameters using Lifshitz theory for lignin and glucomannan interacting with cellulose, titania and calcium carbonate in vacuum, water and hexane. The Hamaker constants for the different combinations of cellulose, lignin and glucomannan in vacuum and water were within in relatively narrow ranges of 35-58 zJ and 8-17 zJ respectively. The estimated Hamaker constants for the interactions of cellulose, lignin and 
from 3 to $19 \mathrm{zJ}$ in water, to being essentially zero for glucomannan interacting with calcium carbonate in hexane. Glucomannan displays significantly smaller Hamaker constants than

3 cellulose and lignin, for the interactions with $\mathrm{TiO}_{2}$ and $\mathrm{CaCO}_{3}$, illustrating that the relative

4 importance of the dispersive forces differs for the different wood components.

5 Keywords: Glucomannan, lignin, cellulose, spectroscopic ellipsometry, dispersion

6 forces, Hamaker constant

\section{Introduction and theoretical background}

8 Wood has been used by mankind from the beginning of civilization as fuel and construction 9 material and has for several centuries also been the major raw material for paper and packaging materials. Novel cellulose-based composites are attracting a large interest in the search for solutions to meet the increasing demand for sustainability of products and production processes and to meet the challenges of decreasing profits in the traditional forest industry. The refined use of wood and wood components in new applications requires a profound understanding of the intrinsic material properties, in order to achieve an optimal utilization of the raw material and efficient modifications to serve the intended purpose. When considering new applications or functionalities for cellulose-containing composites, whether they are used in electronics, pharmaceuticals, construction materials or electrical insulation, the nature of the interactions of the wood-based material with other materials such as polymers (bio- or oil-based), inorganic fillers or solvents is important. The dispersive interactions between the molecular and fibril building blocks strongly influence the processing and also the combined material properties.

The van der Waal (vdW) forces, also known as dispersion forces, play an important role in the behavior of a material in, for example, contact with other materials and are therefore also important for applications of the material (French 2000). Hamaker developed a theoretical description of the van der Waals interactions in macroscopic bodies as a pair-wise summation of the forces between all atoms in the interacting bodies (Hamaker 1937). This summation results in a $\mathrm{vdW}$ interaction free energy inversely proportional to the distance between the bodies. The material response to an excitation by an electromagnetic field at a given frequency is characterized by the complex refractive index, $n(\omega)$, or alternatively by the dielectric function, $\varepsilon(\omega)$, quantities that can be obtained using e.g. spectroscopic ellipsometry (Tompkins and McGahan 1999). The Hamaker constant, which is a measure of the magnitude of dispersion forces for a specific material combination, can be calculated from a few parameters representing the optical response of a material. It is now well established (Parsegian and Ninham 1969; Hough and White 1980; Bergström 1997) how the spectroscopic parameters such as the characteristic frequency, $\omega_{\mathrm{UV}}$, and the adsorption strength $C_{\mathrm{UV}}$, can be obtained from the dielectric data in the UV-visible and IRrange adsorption band.

The vdW interaction free energy per unit area, $V_{v d W}$, between two semi-infinite planar solids separated by a parallel gap, $D$, is expressed as:

$$
V_{v d W}=\frac{A_{H}}{12 \pi D^{2}}
$$


2 This shows the direct proportionality between the Hamaker, $A_{H}$, constant and the van der Waal

3 forces. The non-retarded Hamaker constant $A_{132}$ for two materials 1 and 2 interacting over medium

$4 \quad 3$ is given by (Lifshitz 1956):

$$
A_{132}=\frac{3 k_{B} T}{2} \sum_{m=0}^{\infty} \sum_{s=1}^{\infty} \frac{\left(\Delta_{13} \Delta_{23}\right)^{s}}{s^{3}}
$$

where $k_{B}$ is Boltzmann's constant, $T$ is the absolute temperature and 9

$$
\Delta_{k l}=\frac{\varepsilon_{k}\left(i \xi_{m}\right)-\varepsilon_{l}\left(i \xi_{m}\right)}{\varepsilon_{k}\left(i \xi_{m}\right)+\varepsilon_{l}\left(i \xi_{m}\right)}
$$

12 The prime after the first summation in equation (2) indicates that the contribution of the static term $13(m=0)$ is halved. The dielectric response functions $\varepsilon_{k}\left(\xi_{m}\right)$ and $\varepsilon_{l}\left(\xi_{m}\right)$ of materials $k$ and $l$ are 14 defined as:

$$
\varepsilon\left(i \xi_{m}\right)=1+\sum_{i=1}^{N} \frac{c_{i}}{1+\left(\xi / \omega_{i}\right)^{2}}
$$

where $C_{i}$ is the absorbance strength associated with the absorption band at the characteristic absorption frequency $\omega_{i}$ and $N$ is the number of adsorption bands. These equations are based on the work of Lifshitz (Lifshitz 1956) which has been further elaborated by Ninham and Parsegian (Ninham and Parsegian 1970; Parsegian and Ninham 1969). The calculations need to be evaluated only for the discrete, imaginary frequencies, $i \xi_{m}$, where

$$
\xi_{m}=m\left(\frac{4 \pi^{2} k_{B} T}{h}\right)
$$

where $T$ is the absolute temperature and $h$ is Planck's constant. Since $m$ is an integer; $m=0,1,2$, $3,4, i \xi_{m}$ will be imaginary frequencies of multiples of $2.4 \cdot 10^{14} \mathrm{rad} / \mathrm{s}$ at room temperature. This corresponds to one static term, only a few terms in the infrared and many in the UV-visible range.

29 Hence the dielectric response in the UV-visible range dominates the van der Waals interaction. The determination and accuracy of the spectral parameters in this range is therefore very important. In the present study, the dielectric functions of glucomannan and lignin are represented by one UV and one IR relaxation:

$$
\varepsilon\left(i \xi_{m}\right)=1+\frac{c_{I R}}{1+\left(\xi / \omega_{I R}\right)^{2}}+\frac{c_{U V}}{1+\left(\xi / \omega_{U V}\right)^{2}}
$$

The main components of the wood fibre wall are cellulose, hemicellulose and lignin, of which 37 all contribute to the collective properties of the fibre and the fibre wall. When fibres and fibrils are 38 liberated during chemical and mechanical processing, all three species can be found at their 
external surfaces, interacting with the surrounding media and additives. The Hamaker constants for lignin, hemicellulose and cellulose can provide information regarding the surface interactions in wood fibres which are important for e.g. adhesion, friction, swelling and wetting in paper processing as well as for the resulting behavior of paper products. In nature, the interactions between cellulose, glucomannan and lignin are important for the forces causing aggregation of the cellulose fibrils and are thus important for the structuring and growth of plants and trees. The wetting of wood is also important for the water transport system in plants and for the wood-coating and painting industry. The removal of lignin and hemicellulose decreases the total yield of the wood, so the implications of retaining or removing them for the properties of the final products are of interest. The interfacial properties of cellulose and lignin have been studied with colloidal probe measurements (Notley and Norgren 2006; Notley et al. 2004). Contact angle measurements have also been used to determine the dispersive surface properties of cellulose, lignin and glucomannan (Lee and Luner 1972; Gustafsson et al. 2012; Holmberg et al. 1997; Notley and Norgren 2010). However, the effect of swelling in aqueous media and the influence of H-bonding specificity limits the accuracy when using surface energies to estimate Hamaker constants (Israelachvili 1991). The Hamaker constant for cellulose has been estimated, using spectroscopic ellipsometry and surface force apparatus and applying Lifshitz theory, to $8.4 \cdot 10^{-20} \mathrm{~J}$ in air and $0.86 \cdot 10^{-20} \mathrm{~J}$ in water (Holmberg et al. 1997) and to $5.8 \cdot 10^{-20} \mathrm{~J}$ in air and $0.80 \cdot 10^{-20} \mathrm{~J}$ in water (Bergström et al. 1999). The Hamaker constant of lignin-water-lignin has been estimated to $1.9 \cdot 10^{-22} \mathrm{~J}$ using the relation to the critical coagulation concentration (Norgren et al. 2001). In order to use the Lifshitz equation to estimate the Hamaker constants of lignin and glucomannan, it is necessary to determine spectral data for the respective component.

In this work, the dielectric properties as well as the spectral parameters of films of pure lignin and pure glucomannan have been estimated by spectroscopic ellipsometry. These results have been compared with those obtained in previous studies of cellulose (Bergström et al. 1999) and then used to discuss how each of these components influences the van der Waals interactions in wood and in paper.

\section{Experimental section}

\section{Materials}

Commercially available Kraft lignin from cooking liquor, Indulin AT (MeadWestvaco, Richmond, VA) was purified according to procedures previously described (Norgren et al. 2006). To remove carbohydrates, the lignin was dissolved in an acetone:water mixture (8:2) and stirred for $2 \mathrm{~h}$ at room temperature. The mixture was centrifuged and the residue containing undissolved carbohydrates was removed. The acetone was removed from the suspension through rotor evaporation, water being repetitively added until all acetone was evaporated. The remaining lignin was freeze-dried and the freeze-dried lignin was finally extracted with pentane for $8 \mathrm{~h}$ to remove extractives.

To prepare purified glucomannan, spruce (Picea abies) wood chips obtained from a paper mill in Sweden were cut into $1 \mathrm{~cm}$ wide pieces and mechanically milled into 20 mesh meal (Zhang et 
al. 2011). A total of $50 \mathrm{~g}$ of wood meal was mixed with $300 \mathrm{ml}$ of acetone and incubated overnight to remove the extractives. After filtering to remove the acetone, the wood meal was suspended in $800 \mathrm{ml}$ of water and heated to $75{ }^{\circ} \mathrm{C}$, and $6 \mathrm{~mL}$ of acetic acid (HAc) and $15 \mathrm{~g}$ of sodium chlorite were added in a well-ventilated fume hood to achieve delignification. The addition of HAc and sodium chlorite was repeated seven times at one-hour intervals. After cooling and filtration, the holocellulose thus obtained was thoroughly rinsed with water. Approximately $400 \mathrm{~mL}$ $\mathrm{NaOH} / \mathrm{H}_{3} \mathrm{BO}_{3}(17.5 / 4.0 \%)$ solution was added to the holocellulose in a plastic (polyethylene) bottle and the mixture was shaken overnight under a nitrogen atmosphere. The solution was filtered, and Fehling solution (containing $69.2 \mathrm{gL}^{-1} \mathrm{CuSO}_{4}$ ) was added until no further precipitation of the copper complex was observed. After incubation for 4 hours, the solution was centrifuged (4000 rpm) for $15 \mathrm{~min}$, and the precipitate was washed 4 times with deionised water. The precipitate was subsequently dissolved in $40-60 \mathrm{~mL}$ of $1 \mathrm{M} \mathrm{HCl}$ and two volumes of $96 \%$ cooled $\left(0{ }^{\circ} \mathrm{C}\right)$ ethanol were then added. The solutions were then centrifuged (4000 rpm for $15 \mathrm{~min}$ ) and subsequently washed with $70 \%$ ethanol, $96 \%$ ethanol and a finally with pure ethanol, followed by freeze-drying. The crude glucomannan $(4.6 \mathrm{~g})$ obtained was added to $400 \mathrm{ml}$ of $\mathrm{NaOH} / \mathrm{H}_{3} \mathrm{BO}_{3}$ solution and extracted, precipitated and macerated as described above. The precipitate from the cooled ethanol was washed with pure ethanol until a colourless wash was obtained and then freeze-dried. The purified glucomannan consisted of galactose, glucose and mannose in a ratio of 0.03:1:3.4, with a molecular weight, $M_{\mathrm{w}}$, of $10 \mathrm{kDa}$, and almost free from other polysaccharides, such as xylan $(0.61 \%)$, and metal ions $(<0.2 \%$ ash content).

Polished silicon wafers were purchased from MEMC Electronic Materials (Novara, Italy).

\section{Preparation of lignin and glucomannan films}

Pure lignin and glucomannan films were obtained by spin-coating, using a KW-4A spin-coater (Chemat Technology, Northridge, CA, USA). The solutions for spin-coating were prepared according to Gustafsson et al. (Gustafsson et al. 2012). Lignin and glucomannan, 1 wt\%, were stirred for $24 \mathrm{~h}$ in $\mathrm{NH}_{3}(25 \%)$, at room temperature. The solutions were thereafter filtered through $0.2 \mu \mathrm{m}$ polyethersulfone membrane syringe filters. Spin-coating was performed for $60 \mathrm{~s}$ at 2000 rpm on cleaned and plasma-treated silicon surfaces with native oxide.

\section{Surface characterization}

The surface morphology and surface roughness were investigated by Atomic Force Microscopy (AFM) using a Nanoscope IIIa AFM (Bruker AXS, Santa Barbara, CA, USA) with a type E piezoelectric scanner. The measurements were done in tapping mode using a TAP150 (Bruker, Camarillo, CA, USA) cantilever with a typical spring constant of $5 \mathrm{~N} / \mathrm{m}$. The root mean square surface roughness $\left(R_{\mathrm{q}}\right)$ was obtained by the AFM software (Nanoscope Analysis, Veeco Instruments) and was further averaged over three $1 \mu \mathrm{m}^{2}$ areas. 
monochromated $\mathrm{Al} \mathrm{K}$ source and with an analysis area of $0.3 \times 0.7 \mathrm{~mm}^{2}$. The measurements were

2 performed at Umeå University, Sweden.

\section{Spectroscopic ellipsometry}

5 Measurements in the ultraviolet-visible-near infrared (UV-VIS-NIR) region 245-1690 nm were 6 performed using a variable-angle ellipsometer with dual rotating compensators (RC2, J. A. 7 Woollam Co., Inc.). The measurements were made at and $23{ }^{\circ} \mathrm{C}$ and $48 \% \mathrm{RH}$. The samples in 8 these measurements were the deposited films of lignin and glucomannan on silicon substrates. 9

\section{Calculations of Lifshitz-Hamaker constants}

11 All the calculations correspond to room temperature (298 K). The summation in equation (2) was stopped for $m_{\max }=3000$ and $s_{\max }=4$, after checking the convergence.

\section{Results and discussions}

\section{Surface characterization}

16 AFM measurements showed that the materials were smooth, with a roughness in the sub17 nanometer range (table 1). AFM tapping mode images of glucomannan and lignin model materials 18 are shown in figures $1 \mathrm{a}$ and $\mathrm{b}$, respectively. The glucomannan model films were homogeneous and 19 had a roughness, $R_{\mathrm{q}}$, of $0.29 \mathrm{~nm}$. The lignin model surface had a slightly greater surface roughness 20 of $0.74 \mathrm{~nm}$.

22 Table 1 Film thickness from ellipsometry measurements and root mean square surface roughness, $23 \mathrm{R}_{\mathrm{q}}$, from AFM measurements.

\begin{tabular}{lcc}
\hline Material & Film thickness $[\mathrm{nm}]$ & $R_{\mathrm{q}}[\mathrm{nm}]_{25}^{24}$ \\
\hline Glucomannan & $25.5 \pm 0.5$ & $0.29 \pm 0.01$ \\
Lignin & $40.4 \pm 0.4$ & $0.74 \pm 0.25$ \\
\hline
\end{tabular}



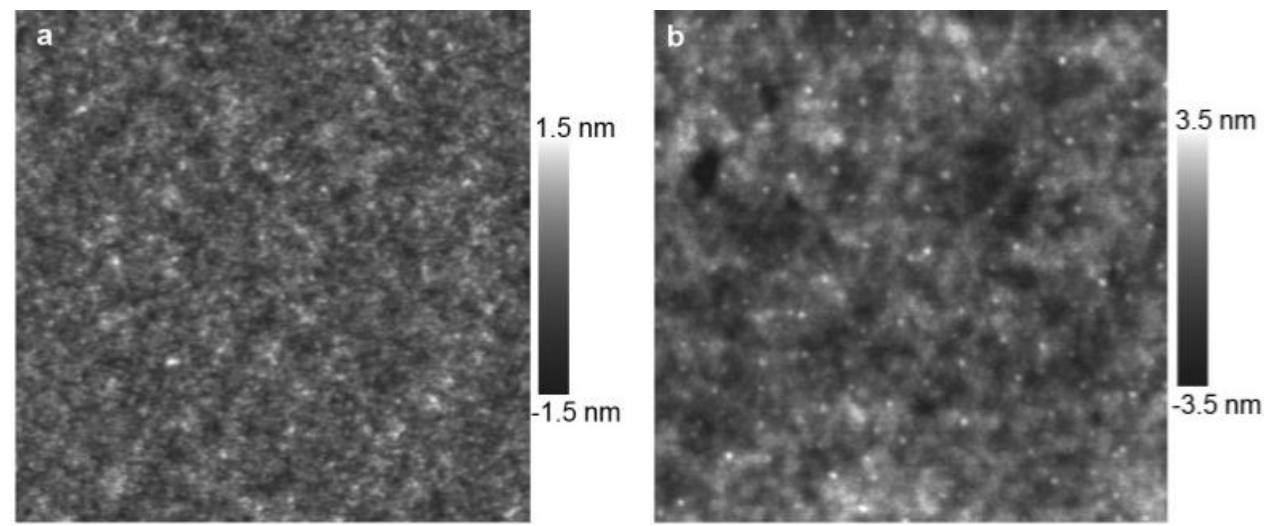

1 Fig. 1 1x1 $\mu \mathrm{m}$ AFM tapping mode images of (a) glucomannan and (b) lignin spin-coated model materials on silicon substrates. The scale bars on the right in the figures correspond to the z-range in the images.

The results of the XPS analysis of the spin-coated films are given in table 2. The O/C ratios,

60.74 and 0.41 for glucomannan and lignin respectively, indicate presence of small amounts of carbohydrate in the lignin sample and extractives and/or lignin in the glucomannan sample (Laine et al. 1994; Carlsson et al. 1995). The theoretical O/C values for hemicellulose and lignin are 0.80 and 0.33 respectively (Gustafsson et al. 2002).

Table 2 XPS data for the atomic surface concentrations of the model films.

\begin{tabular}{|c|c|c|c|c|c|c|}
\hline \multirow[t]{2}{*}{ Material } & \multicolumn{5}{|c|}{ Surface concentration [atomic \%] } & \multirow{2}{*}{$\frac{\text { Atomic ratio }}{\mathrm{O} / \mathrm{C}}$} \\
\hline & $\mathrm{C}$ & $\mathrm{O}$ & $\mathrm{N}$ & $\mathrm{Na}$ & $S$ & \\
\hline Glucomannan & 57.2 & 42.3 & & 0.9 & & 0.74 \\
\hline Lignin & 70.8 & 29.2 & 1.8 & 0.2 & 0.3 & 0.41 \\
\hline
\end{tabular}

\section{Spectroscopic ellipsometry}

The thicknesses of the model films measured by ellipsometry are given in table 1 . The thickness of the glucomannan films were lower than that of the lignin films produced by the same procedure. The glucomannan film, approximately $25 \mathrm{~nm}$ in thickness, was obtained after spin-coating two layers, each according to the described procedure.

Molecular electronic absorption usually consists of a broad absorption band, in contrast to that of a single atom, which absorbs at a discrete absorption frequency. This is due to the presence of a range of vibrational and rotational states. For insulating materials, which have a high band gap, i.e. the energy difference between the highest valence band and the lowest conduction band, the absorption bands are found at the lower wavelengths of the UV-range at 100-200 nm. This is in contrast to semiconducting materials which have an absorption maximum associated with an electronic excitation at higher wavelengths, typically $300-400 \mathrm{~nm}$, corresponding to lower energies (Bergström 1997; Tompkins and McGahan 1999). 

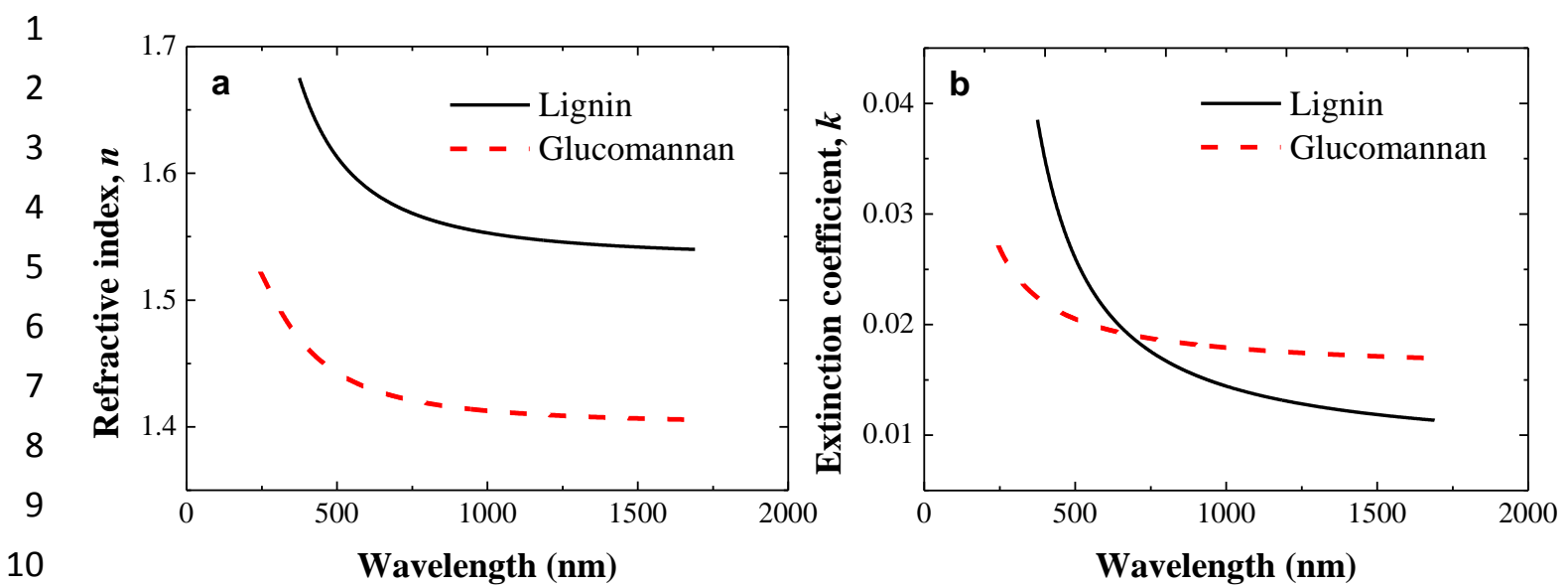

11

12
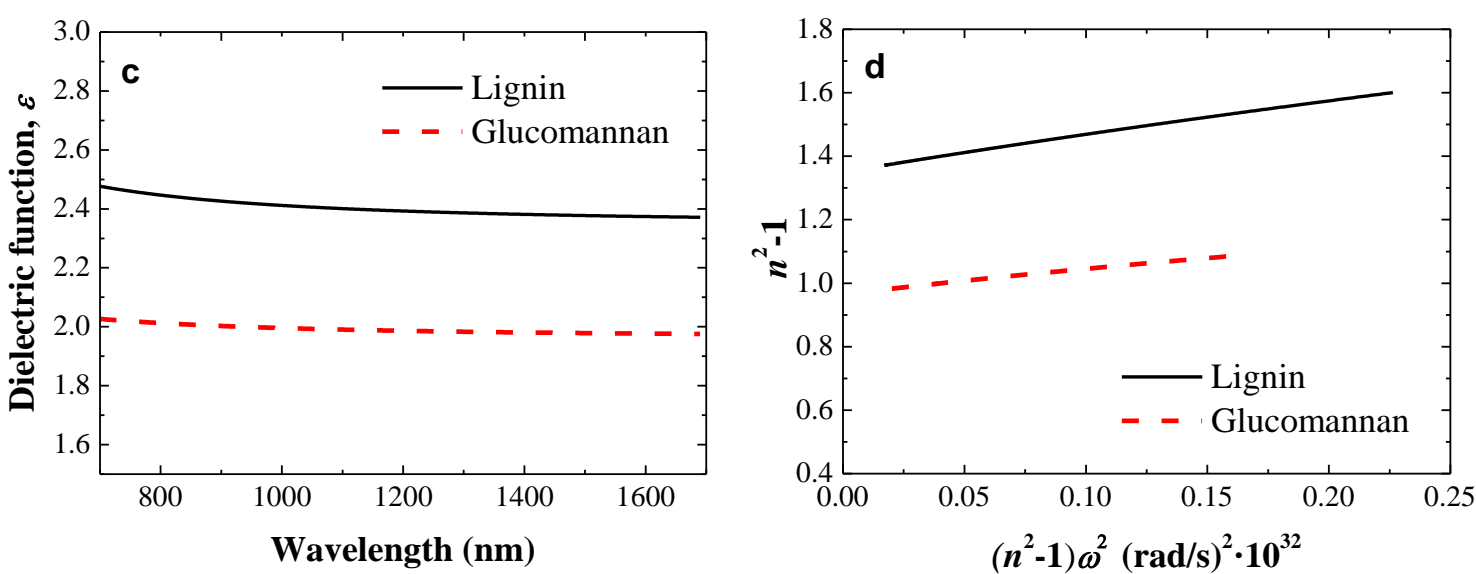

Fig. 2 Data obtained from spectroscopic ellipsometry of lignin (solid line) and glucomannan (dashed line) films. (a) the refractive index and (b) the extinction coefficient versus wavelength, (c) the dielectric function given that $\varepsilon(\omega)=n^{2}$, when $k \approx 0$, and (d) the Cauchy plot with $n^{2}-1$ versus $\left(n^{2}-1\right) \omega^{2}$

Figures $2 \mathrm{a}$ and $\mathrm{b}$ show the wavelength dependence of the refractive index, $n$, and the extinction coefficient, $k$, for glucomannan and lignin in the 300-1700 nm spectral region. The refractive index displays the typical dispersion behavior in this region, decreasing with increasing wavelength. At wavelengths above $700 \mathrm{~nm}$, the extinction coefficient is close to zero $(0.015<k<$ 0.02 ), while $n$ decreases slightly, which is expected for electrically insulating materials. Below 700 $\mathrm{nm}$, the extinction coefficient increases due to an absorption in the lower UV-region. The increase is more rapid for lignin indicating a higher absorption and/or lower absorption frequency. The dielectric function is shown in figure $2 \mathrm{c}$ for the range where $\varepsilon(\omega) \approx n^{2}(\omega)$, which is valid when $k$ is approximately zero at $700-1700 \mathrm{~nm}$. The dielectric function in this range for lignin, that is approximately 2.4, is higher than for glucomannan that is approximately 2.0. According to

37 previously reported data (Bergström et al. 1999), the refractive index and dielectric function for cellulose lie in between those of glucomannan and lignin for the studied frequency range.

The dielectric function is a measure of the polarization and the magnitude of the polarization is related to the length scale of the displacement. At high frequencies (UV, visible and IR ranges) 
major contributions to the dielectric loss originate from electronic (polarization of induced dipoles) and ionic polarization (displacement of atoms in a molecule).

The electronic polarizability of a molecule can be obtained by summarizing the characteristic polarizabilities, $\alpha_{0}$, of its covalent bonds (Israelachvili 1991; Hiemenz 1986). Values for the polarizability of bonds and molecular groups found in the molecular structures of lignin,

glucomannan and cellulose have previously been determined (Denbigh 1940; Israelachvili 1991).

7 Glucomannan and cellulose structures contain aliphatic C-C bonds, C-H bonds, C-O-C bonds and

8 C-O-H bonds, with electronic polarizabilities between 0.48 and 1.28 (with the unit $4 \pi \varepsilon_{0} \cdot 10^{-30} \mathrm{~m}^{3}$ ).

9 Glucomannan and cellulose also contain a small amount of carboxylic groups, which contribute to 10 greater polarizability, $\alpha_{0}(\mathrm{C}=\mathrm{O})$ is $1.36\left(4 \pi \varepsilon_{0} \cdot 10^{-30} \mathrm{~m}^{3}\right)$. Lignin contains aromatic $\mathrm{C}-\mathrm{C}$ bonds, 11 conjugated $\mathrm{C}=\mathrm{C}$ bonds, $\mathrm{C}-\mathrm{O}-\mathrm{C}, \mathrm{C}-\mathrm{H}$ bonds and $\mathrm{C}-\mathrm{O}-\mathrm{H}$ bonds. The conjugated $\mathrm{C}=\mathrm{C}$ bonds have a 12 high electronic polarizability, $1.65\left(4 \pi \varepsilon_{0} \cdot 10^{-30} \mathrm{~m}^{3}\right)$, which contributes to the dielectric function in 13 the wavelength-range investigated. The values of the refractive index and of the dielectric function 14 are also dependent on the density and on moisture content. Figure $2 \mathrm{~d}$ show that lignin has the highest permittivity even though its three dimensional network-like structure should provide a material with lower structural density than the linear structure of cellulose. Reported values of the densities are $1.5 \mathrm{~g} / \mathrm{cm}^{3}$ for cellulose (Hermans et al. 1945) and $1.3 \mathrm{~g} / \mathrm{cm}^{3}$ for Kraft lignin (Hu 2002). The higher permittivity for cellulose than glucomannan could originate from density differences; in this case the branched glucomannan should have the lower structural density of the two. The incorporation of a medium with a higher dielectric constant, e.g. water also increases the polarizability $/ \mathrm{m}^{3}$. It has previously been concluded (Bergström et al. 1999) that the influence of humidity on spectroscopic ellipsometry measurement on cellulose Langmuir-Blodgett films is minor, $\Delta n<0.01$.

\section{Calculations of spectral parameters}

The spectral parameters for UV absorption, $C_{\mathrm{UV}}$, and frequency, $\omega_{\mathrm{UV}}$, can be estimated, for real $\omega$, from equations (7-8), which are valid for when $\varepsilon^{\prime \prime}(\omega)=0$ in the UV-visible region (Hough and White 1980).

In the so-called Cauchy plot, where $n^{2}-1$ is plotted as a function of $\left(n^{2}-1\right) \omega^{2}$, the value of $C_{\mathrm{UV}}$ can be obtained from the intercept and $\omega_{\mathrm{UV}}$ can be derived from the slope.

The spectral parameters from the IR absorption $C_{\mathrm{IR}}$ and $\omega_{\mathrm{IR}}$, can be extracted from IR-spectra and the relation $C_{\mathrm{IR}}=\varepsilon(0)-C_{\mathrm{UV}}-1$. In the present study, the non-retarded Hamaker constant has been calculated for material combinations across vacuum and water. In the case of cellulose, two sets of spectral parameters in the IR-range were used for the calculations (Bergström et al. 1999). 
Figure 2d shows the Cauchy plot based on data from spectroscopic ellipsometry in the 700$1700 \mathrm{~nm}$ range. The spectral parameters obtained are given in table 3. The UV absorption strengths, $C_{\mathrm{UV}}$, obtained were 1.36 for lignin and 0.97 for glucomannan. The absorption frequency, $\omega_{\mathrm{UV}}$, was calculated from the slope to be $0.95 \cdot 10^{16} \mathrm{rad} / \mathrm{s}$ for lignin and $1.14 \cdot 10^{16} \mathrm{rad} / \mathrm{s}$ for glucomannan, which corresponds to wavelengths of approximately $200 \mathrm{~nm}$ and $165 \mathrm{~nm}$ respectively.

The strength of the absorbance in the IR range, $C_{\mathrm{IR}}$, was approximated from the relation: $C_{\mathrm{IR}}=$ $\varepsilon(0)-C_{\mathrm{UV}}-1$ and $\omega_{\mathrm{IR}}$ is defined as the frequency for the major absorption band in the IR range. It was assumed that the static dielectric constants $\varepsilon(0)$ used for the calculations of the IR absorption strengths for all three materials are 7.0. This is in the higher range of previously reported values (Stoops 1934; Heathcote 1998; Ventkateswaran 1965); however the effect of varying the static dielectric constants (individually or simultaneously for the three materials) from 7.0 down to 3.0

13 was studied and showed only small influence on the Hamaker constants and no influence on the 14 tendency observed. The purified glucomannan has its main absorption peak at $1036 \mathrm{~cm}^{-1}$ 15 corresponding to the C-O-C vibration (Zhang et al. 2011), $\omega_{\mathrm{IR}}=2.0 \cdot 10^{14} \mathrm{rad} / \mathrm{s}$. Earlier published 16 data regarding the IR spectra for Indulin AT (lignin) (Fox and McDonald 2010) shows a main IR 17 absorption band at about $1300 \mathrm{~cm}^{-1}$, corresponding to $\omega_{\mathrm{IR}}=2.5 \cdot 10^{14} \mathrm{rad} / \mathrm{s}$.

Table 3 Spectral parameters for lignin, glucomannan and cellulose.

\begin{tabular}{lcccccc}
\hline \multicolumn{1}{c}{ Material } & $C_{\mathrm{UV}}$ & $\begin{array}{c}\omega_{\mathrm{UV}} \\
(\mathrm{rad} / \mathrm{s}) \cdot 10^{16}\end{array}$ & $C_{\mathrm{IR} 1}$ & $C_{\mathrm{IR} 2}$ & $\begin{array}{c}\omega_{\mathrm{IR} 1} \\
(\mathrm{rad} / \mathrm{s}) \cdot 10^{14}\end{array}$ & $\begin{array}{c}\omega_{\mathrm{IR} 2} \\
(\mathrm{rad} / \mathrm{s}) \cdot 10^{14}\end{array}$ \\
\hline Lignin & 1.36 & 0.95 & 4.64 & - & 2.5 & - \\
Glucomannan & 0.97 & 1.14 & 5.03 & - & 2.0 & - \\
Cellulose* & 1.24 & 1.29 & 2.52 & 2.24 & 2.1 & 6.3 \\
\hline
\end{tabular}

The spectroscopic ellipsometry data have so far been expressed using wavelength and 23 frequency. In the UV-range, it is also common to discuss the energy $E_{\mathrm{p}}$, in $\mathrm{eV}$, for photon 24 absorption, corresponding to an excitation of an electron from an occupied orbital to an 25 unoccupied or partially unoccupied orbital. The absorption energy can be approximated from the 26 absorption maximum $\omega_{\mathrm{UV}}$, as $E_{\mathrm{p}} \approx \hbar \omega_{\mathrm{UV}}$ ( $\hbar$ is the reduced Planck constant). The energies of the 27 characteristic absorption frequencies of lignin, glucomannan and cellulose have been 28 approximated on the basis of this relationship and are shown in table 4. According to this 29 approximation, cellulose has the highest energy for the main electronic transitions, $9.1 \mathrm{eV}$, 30 whereas it is $8.0 \mathrm{eV}$ glucomannan and lignin has a value as low as $6.5 \mathrm{eV}$. The characteristic 31 absorption peak for lignin at about $280 \mathrm{~nm}(4.9 \mathrm{eV})$ represents the lowest absorption energy of any 32 of the materials, although the absorbance at this wavelength is relatively low. The high energy for 33 absorption for cellulose is related to its good performance as electrical insulation material which is 34 utilized in several applications (Heathcote 1998). Although lignin is electrically insulating it has 35 interesting charge transfer characteristics (Barsberg et al. 2005; Furman and Lonsky 1988), 
enhanced by quinone presence, that has been recently recognized for the potential use in e.g. energy storage applications (Milczarek and Inganäs 2012).

Table 4 Frequencies, wavelengths and approximated energies associated with absorption maxima

\begin{tabular}{lccrr}
\hline Material & $\begin{array}{c}\omega_{\mathrm{UV}} \\
(\mathrm{rad} / \mathrm{s}) \cdot 10^{16}\end{array}$ & $\begin{array}{c}\lambda \\
(\mathrm{nm})\end{array}$ & \multicolumn{2}{c}{$\begin{array}{c}\text { Photon } \\
\text { energy }(\mathrm{eV})\end{array}$} \\
\hline Lignin & 0.95 & 200 & 6.5 & 8 \\
Glucomannan & 1.14 & 165 & 8.0 & $\begin{array}{r}9 \\
\text { Cellulose* }\end{array}$ \\
\hline
\end{tabular}

\section{Hamaker constants}

The Hamaker constants for various material combinations in water, in vacuum and in hexane were calculated according to equation (2) and are given in table 5. The value of the Hamaker constant for the lignin-water-lignin $\left(A_{\mathrm{lwl}}\right)$ combination was calculated to $A_{\mathrm{lwl}}=1.7 \cdot 10^{-20} \mathrm{~J}$. This is two orders of magnitude greater than the previously reported value $1.9 \cdot 10^{-22} \mathrm{~J}$ (Norgren et al. 2001), where coagulation kinetics were used to estimate the Hamaker constants. This latter way of estimating the Hamaker constant is associated with larger and further reaching approximations than used in present investigation. In vacuum, where the UV-parameters are of larger importance, the differences between the calculated values of the Hamaker constants are larger than in water. In vacuum, the Hamaker constant for glucomannan, $A_{\text {gvg }}, 3.5 \cdot 10^{-20} \mathrm{~J}$ is the lower than for cellulose and lignin in vacuum, and glucomannan also has the lowest $\mathrm{vdW}$ interactions with lignin and cellulose, $A_{\mathrm{lvg}}=4.0 \cdot 10^{-20} \mathrm{~J}$ and $A_{\text {cvg }}=4.5 \cdot 10^{-20} \mathrm{~J}$. Cellulose, on the other hand have the highest Hamaker constant in vacuum, $A_{\mathrm{cvc}}=5.8 \cdot 10^{-20} \mathrm{~J}$, and also the strongest $\mathrm{vdW}$ interactions with glucomannan and lignin $A_{\mathrm{cvg}}=4.5 \cdot 10^{-20} \mathrm{~J}$ and $A_{\mathrm{cvl}}=5.2 \cdot 10^{-20} \mathrm{~J}$. The Hamaker constant for cellulosevacuum-cellulose, $A_{\mathrm{cvc}}=5.8 \cdot 10^{-20} \mathrm{~J}$, is somewhat lower than the previously reported value $8.4 \cdot 10^{-20}$ J calculated from the surface energy (Holmberg et al. 1997). However, using the surface energy of cellulose and other hydrogen-bonding materials to estimate the Hamaker constant underestimates the influence of H-bonding forces (Israelachvili 1991). When vacuum replaces water the van der Waals interactions become stronger, which is most significant for the interaction with $\mathrm{TiO}_{2}$ and $\mathrm{CaCO}_{3}$. The Hamaker constants for lignin, cellulose and glucomannan with the two common paper fillers, $\mathrm{TiO}_{2}$ and $\mathrm{CaCO}_{3}$, as the second material are given in table 5, where the oscillator parameters for $\mathrm{TiO}_{2}$ and $\mathrm{CaCO}_{3}$ are taken from literature (Bergström 1997). The Hamaker constants with $\mathrm{TiO}_{2}$ and $\mathrm{CaCO}_{3}$ in vacuum (dry conditions) show that the dispersive interactions between these fillers and the biopolymers are higher than those between the biopolymers. Hence, the loss in dry strength of paper upon the addition of inorganic filler is not due to a decrease in the

39 dispersive interactions between pulp and filler, but an effect of impaired consolidation. However, 
same range as or lower than the interactions with the biopolymers. The interactions with $\mathrm{CaCO}_{3}$ are low for all combinations in water.

3 Paper is also used in applications with an organic medium which is apolar or has low polarity.

4 The Hamaker constants in hexane with oscillator parameters taken from literature (Hough and

5 White 1980), in table 5, show that the interactions in such a medium are low both between the

6 biopolymers and between the biopolymers and filler. In one case, for glucomannan and $\mathrm{CaCO}_{3}$, the

7 dispersive interactions may even become repulsive as the estimated Hamaker constant is negative

8 (Feiler et al. 2008).

Table 5 Hamaker constants for material combinations in water and vacuum.

\begin{tabular}{llccc}
\hline & & \multicolumn{3}{c}{ Hamaker constant $\left[10^{-20} \mathrm{~J}\right]$} \\
\hline Material 1 & Material 2 & Medium & \\
& & Water & Vacuum & Hexane \\
\hline Lignin & Lignin & 1.7 & 4.6 & 0.7 \\
& Glucomannan & 1.5 & 4.0 & 0.5 \\
& Cellulose & 1.5 & 5.2 & 0.6 \\
& $\mathrm{CaCO}_{3}$ & 0.6 & 6.4 & 0.2 \\
& $\mathrm{TiO}_{2}$ (rutile) & 1.5 & 8.3 & 1.1 \\
\hline \multirow{6}{*}{ Glucomannanan } & $\mathrm{Glucomannan}$ & 1.5 & 3.5 & 0.5 \\
& $\mathrm{Cellulose}$ & 1.2 & 4.5 & 0.4 \\
& $\mathrm{CaCO}_{3}$ & 0.3 & 7.2 & -0.1 \\
& $\mathrm{TiO}_{2}$ (rutile) & 0.7 & 5.6 & 0.3 \\
\hline Cellulose & $\mathrm{Cellulose}$ & 1.4 & 5.8 & 0.6 \\
& $\mathrm{CaCO}_{3}$ & 0.9 & 9.3 & 0.6 \\
& $\mathrm{TiO}_{2}$ (rutile) & 1.9 & 7.4 & 1.6 \\
\hline
\end{tabular}

\section{Concluding remarks}

13 Thin films of purified glucomannan and lignin with a low surface roughness and well-defined

14 thickness have been produced and characterized. Spectroscopic ellipsometry has been used to 15 measure optical properties in order to evaluate the dielectric properties of glucomannan, lignin and 16 cellulose. The similarity in the molecular structures of glucomannan and cellulose is reflected in 17 the results (e.g. values of the absorption frequency and dielectric function). The more complex 18 structure of lignin, with its conjugated bonds, provides a higher polarizability and lower energy for 19 absorption and electronic transitions.

20 The spectral parameters can be used to estimate the vdW interaction forces, and the colloidal 21 stability of material combinations of relevance to wood and wood products. For wood fibres that 22 have undergone delignification and bleaching, and consequently have low surface charges and 23 small repulsive forces, the van der Waals forces become more important and contribute to the 24 adhesive properties. For the material combinations of cellulose, glucomannan and lignin 
interacting with each other, in dry conditions, the strongest interaction is for the cellulose-vacuumcellulose $\left(A_{\mathrm{cvc}}\right)$ combination, and lignin and glucomannan also have their strongest interactions with cellulose in vacuum. In comparison, in wet conditions the strongest interactions for cellulose, glucomannan and lignin, interacting with each other, are those including lignin. Another application of the collected data is an estimation of the critical coagulation concentration, i.e. the solute concentration at which fast aggregation occurs, for the different materials for a given temperature, charge density and Hamaker constant. The colloidal stability of fibril aggregates in water is lowered by the decrease of surface charge density upon removal of lignin, whereas the reduction in vdW interactions with less lignin does have the opposite effect. In a medium with a Hamaker constant close to those of lignin, glucomannan and cellulose, the Hamaker constants and van der Waals interactions will be low.

The spectral data can be used to estimate the interactions of lignin, cellulose and hemicellulose with inorganic or organic fillers. The Hamaker constant for combinations of lignin, cellulose and glucomannan with the inorganic fillers $\mathrm{TiO}_{2}$ and $\mathrm{CaCO}_{3}$ are higher than the interactions with themselves in vacuum. This indicates that any losses in dry strength due to the addition of these fillers are due to a less well consolidated sheet in the presence of filler and not to a reduction of $\mathrm{vdW}$ forces. Under wet conditions, however, the interactions are in the same range or lower between the biopolymers and fillers than between the biopolymers.

\section{Acknowledgement}

This study is part of a project about cellulose based electrical insulation funded by $A B B A B$ and the Swedish Energy Agency through the ELEKTRA program. LB and BF acknowledge support from the Wallenberg Wood Science Center (WWSC) and the Strategic Research Foundation (SSF). Knut and Alice Wallenberg foundation is acknowledged for support to instrumentation. Lars Ödberg and Claire Pitois are gratefully acknowledged for their valuable

\section{References}

Barsberg S, Matousek P, Towrie M (2005) Structural Analysis of Lignin by Resonance Raman Spectroscopy. Macromol Biosci 5:743-752

Bergström L (1997) Hamaker Constant of Inorganic Materials. Adv Colloid Interfac 70:125-169

Bergström L, Stemme S, Dahlfors T, Arwin H, Ödberg L (1999) Spectroscopic Ellipsometry Characterization and Estimation of the Hamaker Constant of Cellulose. Cellulose 6:1-13

Carlsson G, Ström G, Annergren G (1995) Water Sorption and Surface Composition of Untreated or Oxygen Plasma-treated Chemical Pulps. Nord Pulp Pap Res J 10 (1):17-23

Dagastine RR, Prieve DC, White LR (2000) The Dielectric Function for Water and its Application to van der Waal Forces. J Colloid Interf Sci 231:351-358

Denbigh KG (1940) The Polarizabilities of Bonds - I. T Faraday Soc 36:936-948

Feiler AA, Bergström L, Rutland MW (2008) Superlubricity Using Repulsive van der Waals Forces. Langmuir 24:2274-2276

Fox SC, McDonald AG (2010) Chemical and Thermal Characterization of Three Industrial Lignins and their Corresponding Lignin Esters. BioResources 5 (2):990-1009

French RH (2000) Origins and Applications of London Dispersion Forces and Hamaker Constants in Ceramics. J Am Ceram Soc 83 (9):2117-2146

Furman GS, Lonsky WFW (1988) Charge-transfer Complexes in Kraft Lignin. 1. Occurance. J Wood Chem Technol 8:165-189 
Gustafsson E, Johansson E, Pettersson T, Wågberg L (2012) Direct Adhesive Measurements between Wood Biopolymer Model Surfaces. Biomacromolecules

Gustafsson J, Ciovica L, Peltonen J (2002) The Ultrastructure of Spruce Kraft Pulps Studied by Atomic Force Microscopy (AFM) and X-ray Photoelectron Spectroscopy (XPS). Polymer 44

Hamaker HC (1937) The London-van der Waals Attraction between Spherical Particles. Physica IV (10):1058-1072

Heathcote MJ (1998) The J\&P Transformer Book a Practical Tehnology of the Power Transformer. Newnes, Oxford

Hermans PH, Hermans JJ, Vermaas D (1945) Density od Cellulose Fibers. II. Density and Refractivity of Model Filaments. J Polym Sci 1:165-161

Hiemenz PC (1986) Principles of Colloid and Surface Chemistry. 2 edn. New York, Marcel Dekker, Inc.

Holmberg M, Berg J, Stemme S, Ödberg L, Rasmusson J, Claesson P (1997) Surface Force Measurement of Langmuir-Blodgett Cellulose Films. J Colloid Interf Sci 186:369-381

Hough DB, White LR (1980) The Calculation of Hamaker Constants from Lifshitz Theory with Applications to Wetting Phenomena. Adv Colloid Interfac 14:3-41

$\mathrm{Hu}$ TQ (ed) (2002) Chemical Modification, Properties, and Usage of Lignin, s.94. Kluwer Academic/Plenum Publisher, New York

Israelachvili J (1991) Intermolecular \& Surface Forces: Chapter 5 and 11.2 edn. Academic Press Inc., San Diego

Laine J, Stenius P, Carlsson G, Ström G (1994) Surface Characterization of Unbleached Kraft Pulps by Means of ESCA. Cellulose 1 (2):145-160

Lee SB, Luner P (1972) The Wetting and Interfacial Properties of Lignin. Tappi J 55:116-121

Lifshitz EM (1956) The Theory of Molecular Attractive Forces between Solids. Sov Phys 2 (1):73-83

Milczarek G, Inganäs O (2012) Renewable Cathode Materials from Biopolymer/Conjugated Polymer Interpenetrating Networks. Science 335:1468-1471

Ninham BW, Parsegian VA (1970) van der Waals Forces: Special Characteristics in Lipid-water Systems and a General Method of Calculations Based on Lifshitz Theory. Biophys J 10 (7):646-663

Norgren M, Edlund H, Wågberg L, Lindström B, Annergren G (2001) Aggregation of Kraft Lignin Derivatives under Conditions Relevant to the Process, Part I: Phase Behaviour. Colloid Surface A 194:85-96

Norgren M, Notley SM, Majtnerova A, Gellerstedt G (2006) Smooth Model Surfaces from Lignin Derivatives. I. Preparation and Characterization. Langmuir 22 (3):1209-1214

Notley SM, Norgren M (2006) Measurement of Interaction Forces between Lignin and Cellulose as a Function of Aqueous Electrolyte Solution Conditions. Langmuir 22:11199-11204

Notley SM, Norgren M (2010) Surface Energy and Wettability of Spin-Coated Thin Films of Lignin Isolated from Wood. Langmuir 26:5484-5490

Notley SM, Pettersson B, Wågberg L (2004) Direct Measurement of Attractive van der Waals' Forces between Regenerated Cellulose Surfaces in an Aqueous Environment. J Am Chem Soc 126:13930-13931

Parsegian VA, Ninham BW (1969) Application of the Lifshitz Theory to the Calculations of van der Waals Forces Across Thin Lipid Layers. Nature 224:1197-1198

Stoops WN (1934) The Dielectric Properties of Cellulose. J Am Chem Soc 56:1480-1483

Tompkins HG, McGahan WA (1999) Spectroscopic Ellipsometry and Reflectometry. John Wiley \& Sons,

Ventkateswaran A (1965) Formulas for the Dielectric Constant and Dissipation Factor of Mixtures and Their Application to the Cellulose System. J Appl Polym Sci 9:1127-1138

Zhang Y, Li J, Lindström ME, Stephan A, Gatenholm P Spruce Glucomannan: Preparation, Purification, Characterization and Derivatization. In: 16 International Symposium on Wood Fiber and Pulping Chemistry (16 ISWFPC), Tianjin, China, 2011. 